\title{
A!
}

This is an electronic reprint of the original article.

This reprint may differ from the original in pagination and typographic detail.

Kettunen, Markus; Jämsä-Jounela, Sirkka-Liisa

\section{FAULT TOLERANT MPC WITH AN EMBEDDED FDI SYSTEM}

Published in:

IFAC PROCEEDINGS VOLUMES

DOI:

10.3182/20060830-2-SF-4903.00026

Published: 01/01/2006

Document Version

Peer reviewed version

Please cite the original version:

Kettunen, M., \& Jämsä-Jounela, S-L. (2006). FAULT TOLERANT MPC WITH AN EMBEDDED FDI SYSTEM.

IFAC PROCEEDINGS VOLUMES, 39(14), 143-148. https://doi.org/10.3182/20060830-2-SF-4903.00026

This material is protected by copyright and other intellectual property rights, and duplication or sale of all or part of any of the repository collections is not permitted, except that material may be duplicated by you for your research use or educational purposes in electronic or print form. You must obtain permission for any other use. Electronic or print copies may not be offered, whether for sale or otherwise to anyone who is not an authorised user. 


\title{
FAULT TOLERANT MPC WITH AN EMBEDDED FDI SYSTEM
}

\author{
Kettunen, M. and Jämsä-Jounela S-L. \\ Aalto University \\ Laboratory of Process Control and Automation \\ Kemistintie 1, FIN-02150 Espoo, FINLAND
}

\begin{abstract}
The fault detection and isolation (FDI) in industrial processes has been under an active study during the last decade, but fault tolerant control applications relying to the traditional FDI methods have been scarce. In this paper a fault tolerant model predictive controller (MPC) with an embedded FDI system is developed for controlling a simulated heavy oil fractionator process, the Shell Control Problem (SCP). Two different kinds of FDI systems are used for achieving the fault tolerance in co-operation with MPC: system based on Principal Component Analysis (PCA) and Partial Least Squares (PLS) and a system based on Subspace Model Identification (SMI). The effectiveness of selected methods was successfully tested by introducing bias and drift faults to simulated process measurements. Finally the results are presented and discussed. Copyright (C) 2006 IFAC
\end{abstract}

Keywords: FTC, FDI, PCA, PLS, SMI, MPC, fault tolerance, simulation, shell control problem.

\section{INTRODUCTION}

During the last decades, fault detection and isolation (FDI) as well as model predictive control (MPC) have been one of the most researched areas in the control field, especially in the process industries. In addition, MPC is one of the most implemented advanced control methods in the chemical industries as McAvoy et al. (2002) have stated in their milestone report. The reason for such popularity has mainly been the demand for efficient and reliable control systems. This demand derives from the need to improve quality of the end products, to increase profits and to ensure a reliable, safer way to run the industrial processes.

The fault diagnosis methods are traditionally divided to process history based methods and methods based on first principle models. Most relevant data-driven methods currently are based on the principal component analysis (PCA) and the partial least squares (PLS). Dynamical variants of the basic methods have been developed (Ku et al., 1995, Chen et al., 1998) and also recursive versions (Qin, 1998, Li et al., 2000). The basic assumption with the static models is that the dynamic characteristics of the process are not changing. Real processes usually have strong dynamic changes, often affecting the accuracy of the static methods. The dynamic behaviour of the process can be captured from process history data by using subspace model identification (SMI) methods. During the last decade a number of different approaches have been proposed for the SMI; canonical variate analysis (Larimore, 1990), N4SID (van Overschee and de Moor, 1994), MOESP (Verhaegen, 1994) and a PCA-based approach by Wang and Qin (2002). The successful FDI studies for dearomatization process by Komulainen et al. (2004) and Vermasvuori et al. (2005) have lead to a question if the FDI could be utilized to enable more efficient control of an industrial process.

Various modifications have been made to the MPC formulation during the past decade especially in order to solve the problems related to nonlinear process models. The MPC has been related to fault tolerant control schemes, mainly due to the flexibility and some inherent resistance to the process disturbances and faults. For instance, Pranatyasto and Qin (2001) have been using MPC as part of their FTC design. 
Fault Tolerant Control (FTC) attempts to enhance the availability of a plant by using the measurements and knowledge of the plant model to improve performance and fault-tolerance of the control system. So far the industrial applications on the field have been scarce, but currently there are several research projects that are aimed at creating an effective, reliable and fault tolerant control system to be used in the process industries. Traditionally the FTC strategies have been applied to simple control systems, but recently the research has been increased in developing FTC systems based on MPC systems. For instance, Pranatyasto \& Qin (2001) have been studying a PCA-based FTC system controlling a simulated fluid catalytic cracking (FCC) unit. Also Prakash et al. (2002) developed fault tolerant control system (FTCS) based on generalized likelihood ratio (GLR) and a standard MPC controller. This FTC system was then applied to a simulated nonisothermal continuous stirred tank reactor (CSTR) system. Later on Patwardhan et al. (2005) improved the system developed by Prakash et al. (2002) and applied it to a laboratory-scale continuous stirred tank heater (CSTH) system and to a simulated heavy oil fractionator process, shell control problem (SCP). In this paper the presented FTC system is based on PCA/PLS and SMI in contrast to the GLR-based system presented in the study of Patwardhan et al. (2005).

This paper is organized as follows: in Chapter 2 the target process is introduced along with the sensor faults. Chapter 3 describes the structure of the FTC. Chapter 4 describes the training of the FDI system and description of the MPC. Chapter 5 contains the results and a discussion. Chapter 6 ends the paper with conclusions.

\section{DESCRIPTION OF THE SHELL HEAVY OIL FRACTIONATOR}

Heavy oil fractionators are used in oil refining industry for initially fractionating the crude oil to different product draws by cooling down the mixedphase oil feed. In a real oil refinery there are several fractionators in series fractionating different products from different product draws. The simulated process contains one reactor section, three heat exchangers, one side stripper, one product feed and three product draws. The process model used in the study was originally presented in 1987 to serve as a standard performance test for new control strategies. The process model is known as the Shell Control Problem by Prett and Morari (1987). The target process is described in Fig.1.

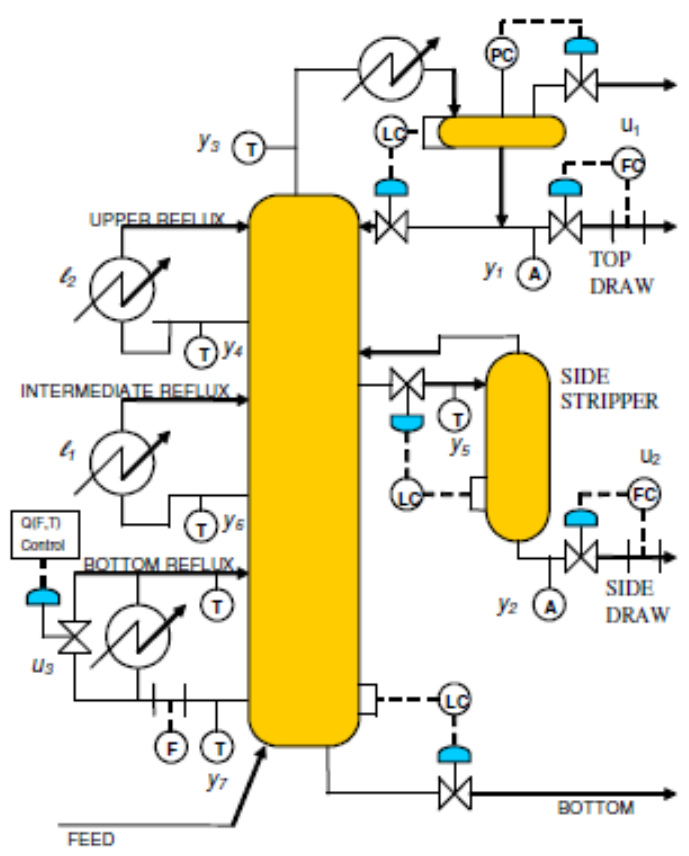

Fig. 1. The Shell Control Problem by Prett and Morari (1987) 
Hot, mixed-phase oil is fed to the unit and cooled down using reflux flows along the fractionator. The different fractions of the oil are divided to product flows leaving the fractionator in different parts of the unit. These reflux flows enable the separation procedure in the fractionator. The heat requirement of this system varies, because the streams are reboiled in other parts of the plant.

The bottom heat reflux loop of the fractionator has an enthalpy controller, regulating the heat removal in the loop by adjusting the creation of the steam. For the purposes of controlling the column, the bottom loop heat duty is used as a manipulated variable (MV). The temperatures of the other two regulating streams are treated as measured disturbances (MD). Finally, for the top and the side draws, the product specifications are determined by operating requirements and economics. In addition there is an operating constraint for the temperature of the bottom draw. The gaseous stream in the feed provides all the required heat for the process. The analyzer outputs $y_{1}$ and $y_{2}$ and the bottom reflux temperature $y_{7}$ are considered as controlled variables $(\mathrm{CV})$.

Heavy oil fractionators are in crucial position in the oil refineries since the purpose of the columns is to produce raw material for the entire refinery. Normal production rates are high, which means that the other parts of the process are dependent of the performance of the fractionators. Faults in the measurement devices or analyzers might cause large economical losses. It is therefore important that the measurement faults in the fractionator measurements are detected and countered as soon as possible.

Constraints for the input and output variables and the variable rates are given in the model description by Prett and Morari (1987). Also the control objectives are given in the description, but the main purpose of this study is to observe and prevent the effect of faults in the process measurements.

\section{THE STRUCTURE OF THE FDI/FTC SYSTEM}

The FTC system developed in the study is constructed of two parts: the FDI-system for detecting and measuring the fault magnitude and the supervisory part for carrying out the necessary actions to prevent the effects of the fault. Several approaches are used for the FDI/FTC: a model based residual evaluation, a system using analytical redundancy and subspace model identified (SMI) model and a PCA/PLS-based evaluator. The PCA is using the standard formulation and the PLS is using the NIPALS algorithm developed by Wold (1975). The SMI algorithm used in the study is a simplified version of the method, developed by Hyötyniemi (2001). The evaluator is using two different kinds of approaches: a measurement signal reconstruction, where the faulty signal is compensated with a correction value and a reference trajectory matching, where the correction is done by changing the MPC reference trajectory. The alternative FTC strategies are presented in the Fig. 2, Fig. 3 and Fig. 4.

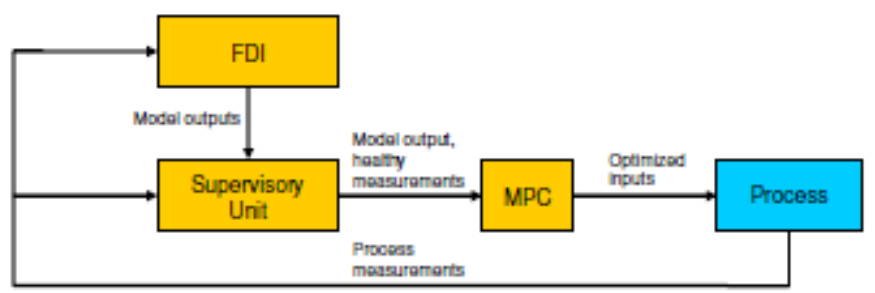

Fig. 2. The structure of the SMI-based FTC system using residual evaluation 


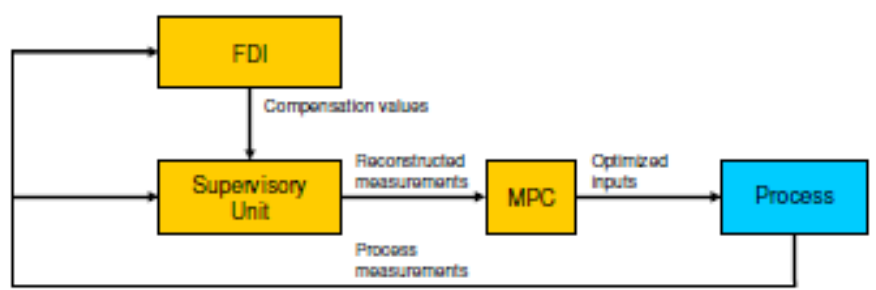

Fig. 3. The structure of the PCA/PLS-based FTC system using measurement signal reconstruction

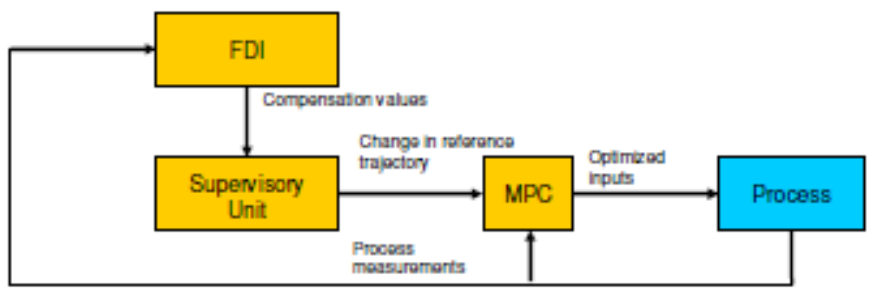

Fig. 4. The structure of the PCA/PLS-based FTC system using reference trajectory matching

\section{TRAINING OF THE FAULT DETECTION AND ISOLATION MODELS AND MPC}

For the FDI training data was created using the original process model under closed-loop control. Closedloop data was used, since the MPC would affect the fault detection procedure and thus the effect of the controller should be included in the FDI models. Also, according to Pranatyasto and Qin (2001), it is more favourable to use closed-loop training data for PCA training when designing FTC system for process with an MPC. In general, the training data was selected to be such that it would contain as much variance as possible to capture the behaviour of the process.

All output and manipulated variables were used in the creation of the FDI models. The output variables were: analyzer outputs $y_{1}$ and $y_{2}$ and temperature measurements $y_{3}, y_{4}, y_{5}, y_{6}$ and $y_{7}$. The manipulated variables were the top draw flow $u_{1}$, the side draw flow $u_{2}$ and bottom reflux flow $u_{3}$.

\subsection{FDI training}

Principal Component Analysis. A PCA model was formed using seven process measurements. In the final model $96 \%$ of the total variance was captured by using three PC's. For FDI purposes, squared prediction error (SPE) and Hotelling T2 limits were calculated using $50 \%$ confidence in both cases. The SPE limit was formed by using the left-out PC's with the method by Jackson (1979). The Hotelling T2 limit was calculated using the standard T2-formulation.

Partial Least Squares. For PLS, the data used for training was formed using less variance as with the PCA, since this approach gave the best result in terms of fault detection and isolation. The long process delays have effect on the PLS behaviour, especially when the there are rapid changes in the process. The PLS latent variables were calculated using the NIPALS algorithm by Wold (1975). In the final model there were four latent variables (LV) which captured about $96 \%$ of input variance and about $72 \%$ of the output variance.

Subspace Model Identification. The training data used for the SMI was same set of data that was used with the PCA. When creating the state-space models, the order of the model was reduced from 35 th order model to 10th order model for optimization purposes. 


\subsection{MPC}

The MPC used a 10th order reduced model, which had almost the same input-output behaviour as the full, perfect 35th order model of the plant. Due to this fact, no robustness problems were encountered with the MPC. The weights on the manipulated variable rates were set to 100 to prevent the effects of the rapid changes caused by the FTC system. Weights for the controlled variables were set to 1 and for the manipulated variables 0.1 .

\section{TESTING, RESULTS AND DISCUSSION}

The evaluation of developed FTC systems was carried out with a simulated data set. Bias and drift faults were introduced to the simulated measurements during the simulation. Whole data set consisted of 800 minutes of simulated process data including measurement errors.

\subsection{Faults}

Two different kinds of faults are common in oil refining process analyzers and sensors: abrupt bias faults and slowly increasing or decreasing drift faults. Bias faults can be caused by some contamination of the analyzer sample. The drift faults are usually caused by a slow accumulation of substance into the sensors or analyzers. In this study, these two fault types are introduced to simulated process measurements. In the test setting, a positive bias fault with a magnitude of 0.9 and a positive drift fault with a target value of 0.9 were introduced to the measurement yl at time 100 minutes and lasted for 200 minutes after which the fault was removed from the measurements.

\subsection{The performance of the FTC systems}

First, the FTC system based on PCA and PLS was tested on the simulated data set. Because the SPE is usually more sensitive to unexpected disturbances than the Hotelling T2, it was decided that the SPE would solely be used as a fault detection index in this study. Hotelling T2 index was used for reference and verification purposes.

The PCA/PLS FTC system was immediately able to detect the bias fault in the process measurement $y_{1}$ and the fault was quickly compensated for. The fault had almost no effect at all to the performance of the process. The drift was detected later than the bias fault, but was also detected and quickly compensated to prevent further effect to the process. The SPE index and Hotelling T2 index in the case of the bias fault are presented in Fig. 5 and for the drift fault in Fig. 6. As it is seen from these figures, the calculated limit in the Hotelling T2 is much higher and thus unable to detect the fault. In both cases the faults are detected at ease with the SPE index. The effect of the fault to the process measurement $y_{1}$ and the compensated measurement signal are presented in the Fig. 7 for the bias fault and in Fig. 8 for the drift fault.

After detecting a fault in the system, a fault isolation procedure based on SPE contribution plots was engaged. As an example, in the case of a bias fault a contribution plot for time step $t=240$ is presented in the Fig. 9. In the contribution plot, the measurement $y_{1}$ is classified as being faulty as can be clearly seen from the figure. The fault identification was carried out by using an iterative procedure based on the SPE score.

Root mean square of prediction error (RMSEP) index was calculated using PLS latent variables, but was used for reference purposes. The RMSEP index was rather sensitive to the different fault types and the faulty signals clearly stand out of the healthy signal values, as it is seen from Fig. 10 for the bias fault and from Fig. 11 for the drift fault. 

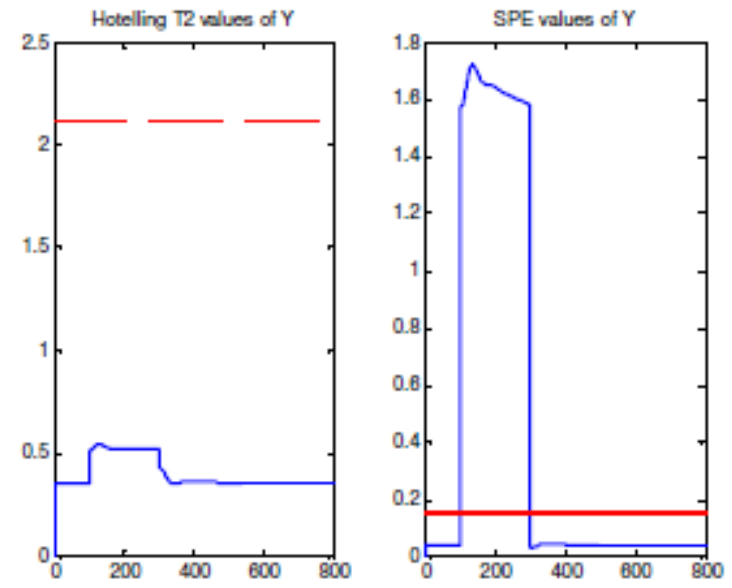

Fig. 5. The Hotelling T2 and SPE indices and limits for the bias-shaped fault in the measurement $y_{1}$
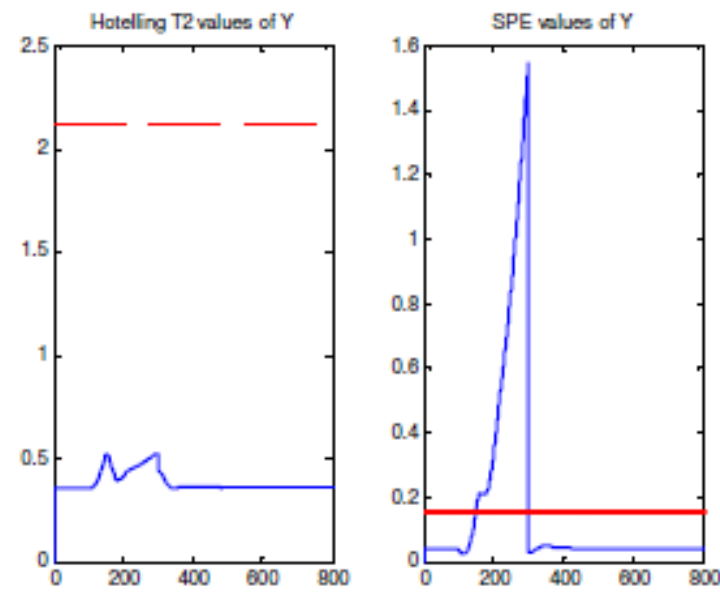

Fig. 6. The Hotelling T2 and SPE indices and limits for the bias-shaped fault in the measurement $y_{1}$

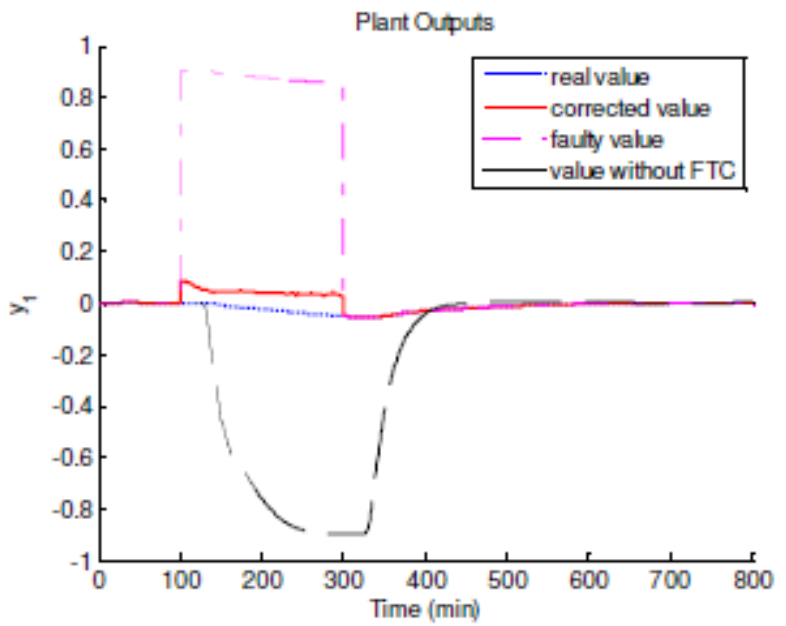

Fig. 7. The effect of the bias fault in the process measurement $y_{1}$ 


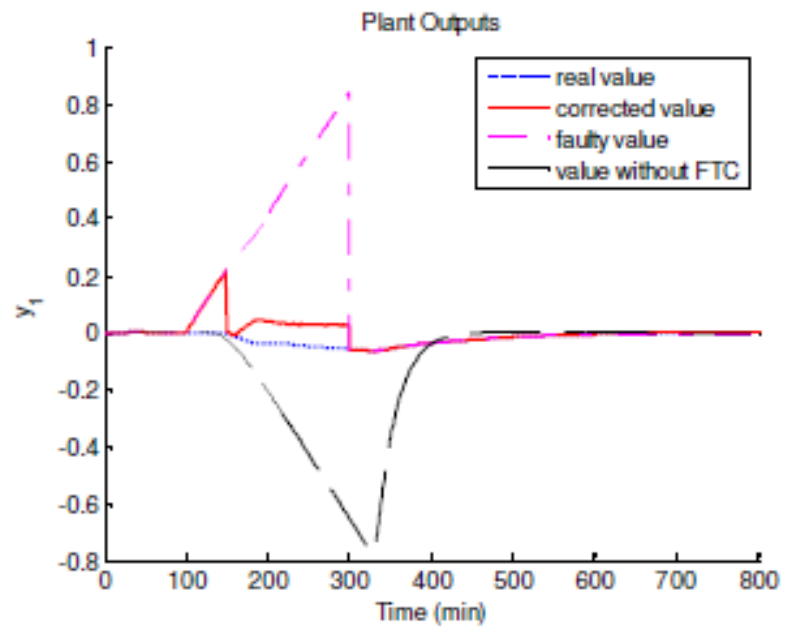

Fig. 8. The effect of the drift fault in the process measurement $y_{1}$

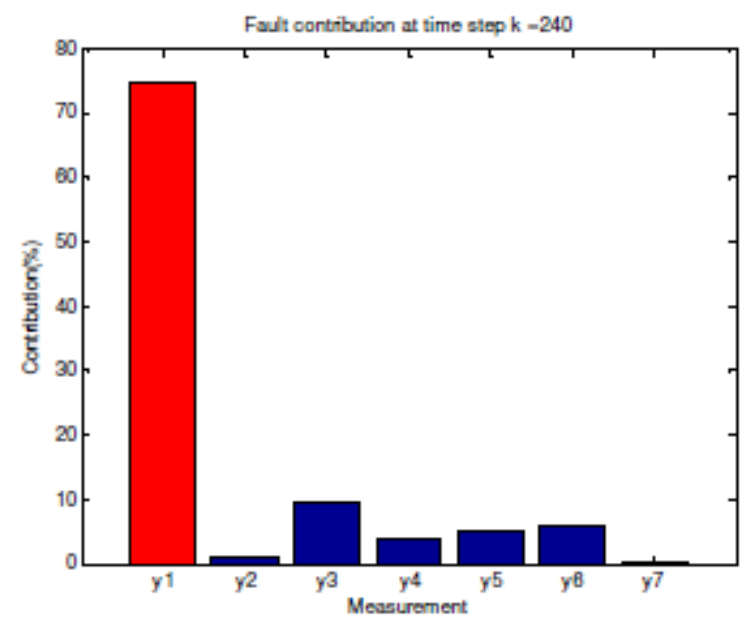

Fig. 9. The SPE contribution of different measurements to the SPE value at time step $t=240$ when a bias fault is present in the output $y_{1}$

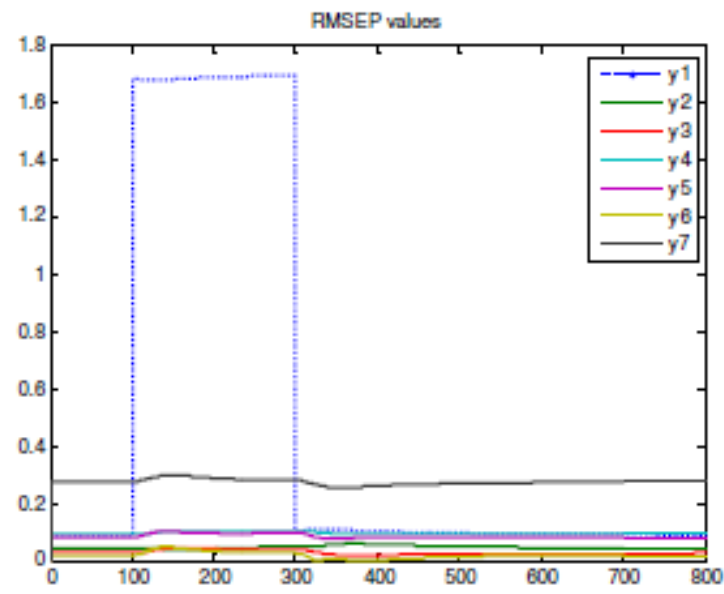

Fig. 10. The RMSEP values for the measurements in the vase of a bias fault in output $y_{1}$ 


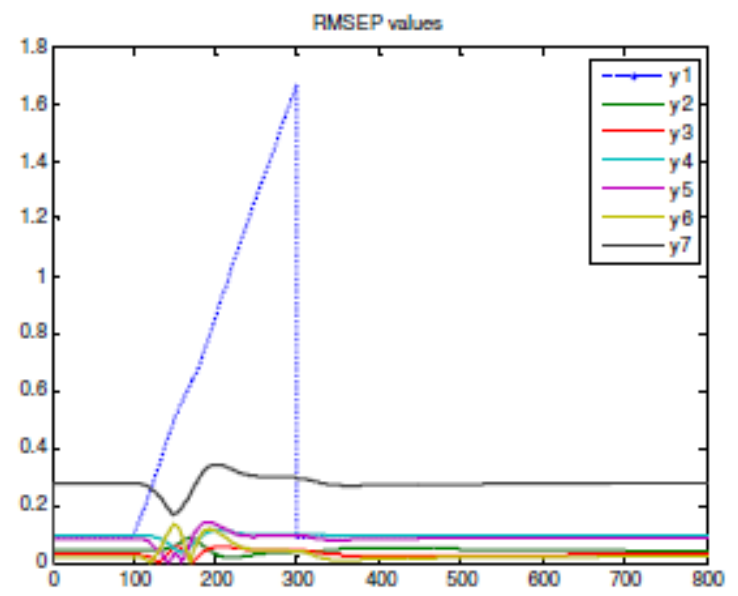

Fig. 11. The RMSEP values for the measurements in the case of a drift fault in output $y_{1}$

Next, the SMI method was tested by using a model based analytical redundancy setting with a model obtained with SMI. By comparing the model outputs with the measurement outputs a residual value was achieved at each time step. If the residual value was higher than predefined limit, a fault was detected and isolated to that specific measurement. When a fault was present in one of the measurements, the model output was used instead of measurement value until the fault had ended and the residual returned under the limit. The performance of the SMI-based system was tested using the bias and drift faults. In the bias case, Fig. 12, the detection and fault compensation works really well, and almost no effect is caused to the process by the fault. In the case of drift fault, the performance is also good as can be seen from Fig. 13.

\subsection{Analysis of the results and discussion}

Based on the results presented in the previous sections, the faults introduced to the system were effectively detected, isolated and compensated with the presented FTC systems. Especially the PCA/PLS based FTC system was working efficiently and the fault detection and isolation rate was good. Also the SMI based analytical redundancy provided good results; the faults were efficiently detected and countered. The results clearly indicate that all the presented methods have potential to be used as effective FDI systems for real industrial processes.

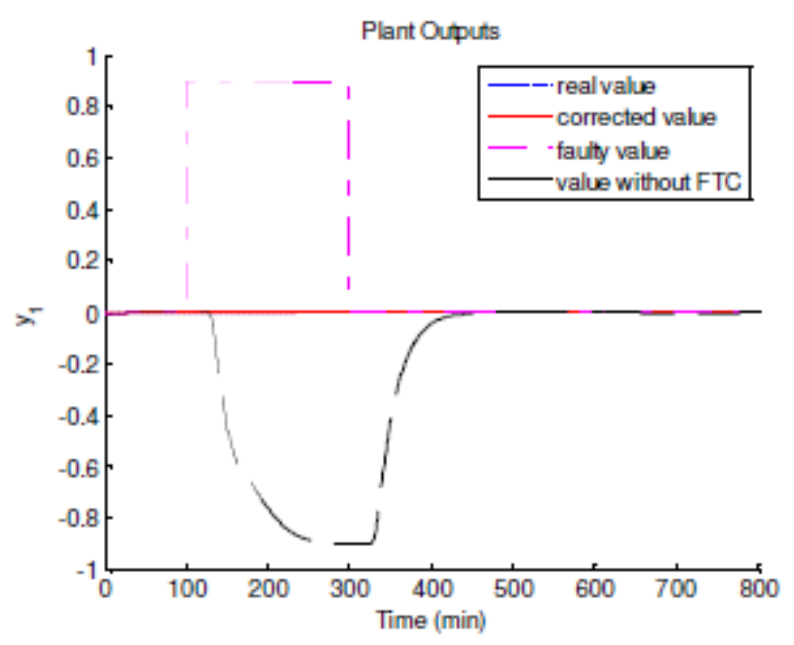

Fig. 12. The performance of the SMI-based FTC system in the case of the bias fault in output $y_{1}$ 


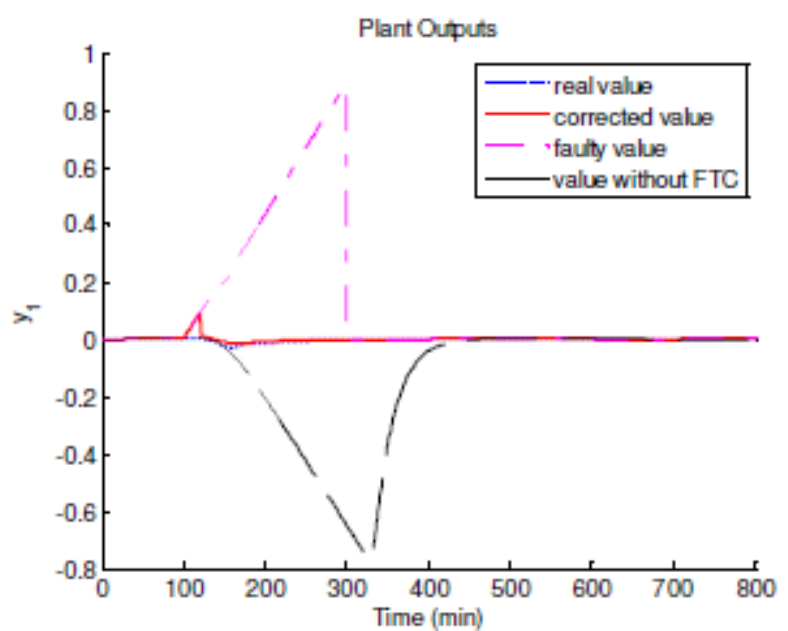

Fig. 13. The performance of the SMI-based FTC system in the case of the drift fault in output $y_{1}$

\section{CONCLUSIONS}

In this paper, PCA, PLS and SMI methods were used for fault detection, isolation and identification purposes to achieve fault tolerance with MPC. Two FTC systems based on these methods were successfully implemented for controlling a simulated heavy oil fractionator. Based on the results, the methods proved to be effective and the FTC systems were able to counter the different faults in the simulated process measurements. In the PCA/PLS based system the use of SPE as a fault detection index was a good choice, because the resolution of the method was better than with the traditionally used Hotelling T2, which was unable to detect faults in the test setting. As a result, the fault detection was working very well in all fault cases. The RMSEP index based on PLS latent variables also provided good results and is definitely a promising addition to a fault-tolerant control system design. The identified SMI model described also the process well and was able to detect the possible faults in the measurements. It is worth noting that the data used in the study was simulated; therefore the results are probably better than they would have been if real process data would have been used instead.

\section{REFERENCES}

Chen, G., T.J. McAvoy and M.J. Piovoso (1998). A multivariate statistical controller for online quality improvement, Journal of Process Control, 8, pp. 139-149.

Hyötyniemi, H. (2001). Multivariate regression - Techniques and tools, Helsinki University of Technology internal report, 125, Helsinki, 159 p.

Jackson, J.E. (1979). Control Procedures for Residuals Associated With Principal Component Analysis, Technometrics, 21, pp. 341-349.

$\mathrm{Ku}$, W., R.H. Storer and C. Georgakis (1995). Disturbance detection and isolation by dynamic principal component analysis. Chemometrics and Intelligent Laboratory Systems, 30, pp.179-196.

Komulainen, T., M. Sourander, S-L. Jämsä-Jounela (2004). An online application of dynamic PLS to a dearomatization process. Computers \&Chemical Engineering, 28, pp. 2611-2619.

Larimore, W. (1990). Canonical variate analysis in identification, filtering and adaptive control. Proceedings of 29th IEEE conference on Decicion and Control, Honolulum Hawaii, pp. 596-604.

Li, W., H.H. Yue, S. Valle-Cervantes and S.J. Qin (2000). Recursive PCA for adaptive process monitoring, Journal of Process Control, 10, pp. 471-486.

McAvoy, T., S-L. Jämsä-Jounela, R. Patton and C. Georgakis (2002). IFAC 2002 Milestone Report on Industrial Applications, 15th Triennial World Congress of the International Federation of Automatic 
Control, Barcelona, Spain, 21th-26 July, 2002 plenary papers, survey papers and milestones, pp. 227232.

Patwardhan, S.C., S. Manuja, S. Narasimhan and S.L. Shah (2006). From data to diagnosis and control using generalized orthonormal basis filters. Part II: Model predictive and fault tolerant control, Journal of Process Control, 16, pp. 157-175.

Prakash, J., S.C. Patwardhan and Narasimhan, S.A. (2002). Supervisory Approach to Fault-Tolerant Control of Linear Multivariable Systems, Ind. Eng. Chem. Res., 41, pp. 2270-2281.

Pranatyasto, T.N. and S.J. Qin (2001). Sensor validation and process fault diagnosis for FCC units under MPC feedback, Control Engineering Practice, 9, pp. 877-888.

Prett, D.M. and M. Morari (1987). Shell Process Control Workshop. Butterworth Publishers, Stoneham, $369 \mathrm{p}$.

Qin, S.J. (1998). Recursive PLS algorithms for adaptive data modelling, Computers and Chemical Engineering, 22, pp. 503-514.

Van Overschee, P. and B. de Moor (1994). N4SID: Subspace algorithms for the identification of combined deterministic-stochastic systems, Automatica, 30, pp.75-93.

Verhaegen, M. (1994). Identification of the deterministic part of MIMO state space models given in innovations form from input-output data, Automatica, 30, pp. 61-74.

Vermasvuori, M., N. Vatanski and S-L. Jämsä- Jounela (2005). Data-based fault detection of the online analysers in a dearomatisation process, Proceedings of 1st NeCST workshop on Networked Control Systems \& Fault Tolerant Control, pp.219-224.

Wang, J. and S.J. Qin (2002) A new subspace identification approach based on principal component analysis, Journal of Process Control, 12, pp. 841-855.

Wold, H. (1975). Path models with latent variables: The NIPALS approach, Quantitative Sociology: International perspective on mathematical and statistical model building, pp. 307-357.

Ying, C-M. and B. Joseph (1999). Performance and Stability Analysis of LP-MPC and QP-MPC Cascade Control Systems, AIChE Journal, 45, pp. 1521-1532. 